\title{
Research on Multi-element Fusion of Equipment Fault Monitoring Based on Evidence Theory
}

\author{
ZHOU Quan ${ }^{1}$, LIU Lan ${ }^{2}$,CHENG Hao ${ }^{1}$, FU Mian ${ }^{1}$ \\ ${ }^{1}$ School of Business, Anhui Xinhua University, 230031, China \\ ${ }^{2}$ School of Maoyin Finance, Anhui Finance \& Trade Vocational College, 230031, China
}

\begin{abstract}
The multi-information fusion method of health monitoring based on evidence theory is used to study the problem of equipment fault diagnosis. The multi-information of fault monitoring is fused by the evidence theory and the reliability of the relevant evidence can be judged according to the ambiguity and uncertainty of the fault monitoring signal. Also it can determine the importance and reliability of the evidence from different sources. The data from multi-information fusion has higher reliability and accuracy which provides more reliable data for fault diagnosis.
\end{abstract}

\section{Analysis of equipment fault monitoring performance signal}

The data of equipment fault monitoring performance signal is essentially a kind of nonstationary time series data (signal) mixed with strong random noise, the data abnormity mainly shows as data point mutation (sporadic data abnormity, such as peak pulse) and data trend Mutation (large data abnormity). The typical characteristics of time series data of equipment fault monitoring performance parameters are analyzed, and the data must be preprocessed before further processing or analyzing.

\section{Identification and reconstruction of} signal abrupt change based on modulus maximum curve of continuous wavelet transform

The wavelet basis function can be obtained by setting $\psi(t)$ as a basic wavelet

$$
\psi_{a, \tau}(t)=\frac{1}{\sqrt{a}} \psi\left(\frac{t-\tau}{a}\right)
$$

In the middle of the ceremony $a>0$, as Scale factor, $\tau$ is as Translation factor, $1 / \sqrt{a}$ has the function to keep the wavelet basis functions have equal energy. Given the square integrable signal $x(t)$, I. E $x(t) \in L^{2}(R)$, then the Continuous Wavelet Transform of $x(t)$ (CWT) is Defined as

$W T_{x(t)}(a, \tau)=\int_{-\infty}^{\infty} x(t) \psi_{a, \tau}^{*}(t) d t=\frac{1}{\sqrt{a}} \int_{-\infty}^{\infty} x(t) \psi^{*}\left(\frac{t-\tau}{a}\right) d t$

In the expression, $*$ denotes conjugation.

The inverse wavelet transform is given by the following theorem:

Theorem 1 Aassume $x(t), \psi(t) \in L^{2}(R)$, $\Psi(\omega)$ is the Fourier transform of the wavelet $\psi(t)$, if the wavelet $\psi(t)$ is satisfied

$$
c_{\psi}=\int_{0}^{\infty} \frac{|\Psi(\omega)|^{2}}{\omega} d \omega<\infty
$$

Then $x(t)$ can be inversed by its wavelet Transform $W T_{x(t)}(a, \tau)$, I. E

$$
\begin{aligned}
x(t) & =\frac{1}{c_{\psi}} \int_{0}^{\infty} \int_{-\infty}^{\infty} W T_{x(t)}(a, \tau) \psi_{a, \tau}(t) \frac{d \tau d a}{a^{2}} \\
& =\frac{1}{c_{\psi}} \int_{0}^{\infty} \int_{-\infty}^{\infty} W T_{x(t)}(a, \tau) \frac{1}{\sqrt{a}} \psi\left(\frac{t-\tau}{a}\right) \frac{d \tau d a}{a^{2}}
\end{aligned}
$$

Formula (3) is called the admissibility condition of wavelets, from which it can be inferred that the basic wavelets should at least satisfy $\left.\Psi(\omega)\right|_{\omega=0}=0$. 
Because wavelet transform and inverse transform have many kinds of definition forms such as inner product, convolution and so on, and the methods used in numerical processing are different, so there are many kinds of realization algorithms. In this project, the continuous wavelet transform formula (2) and its Inverse Transform Formula (4) are transformed into frequency domain by discrete Fourier transform and inverse transform to avoid convolution computation in time domain, thus improving the operation speed.

The Fourier transform of $x(t)$ is $X(\omega)$, the Fourier transform of $\psi(t)$ is $\Psi(\omega)$, and by the translational and similar properties of the Fourier Transform, the Fourier transform of $\psi_{a, \tau}(t)$ is

$$
\Psi_{a, \tau}(\omega)=\sqrt{a} \Psi(a \omega) e^{-i \omega \tau}
$$

So you can get $\Psi_{a, \tau}^{*}(\omega)=\sqrt{a} \Psi^{*}(a \omega) e^{i \omega \tau}$; by Parseval's Theorem, the expression of formula (2) in frequency domain is

$$
W T_{x(t)}(a, \tau)=\frac{1}{\sqrt{a}} \int_{-\infty}^{\infty} x(t) \psi^{*}\left(\frac{t-\tau}{a}\right) d t=\frac{\sqrt{a}}{2 \pi} \int_{-\infty}^{\infty} X(\omega) \Psi^{*}(a \omega) e^{i \omega \tau} d \omega
$$

Similarly, if the wavelet transform $W T_{x(t)}(a, \tau)$ in formula (4) is also treated as a signal, the inverse wavelet transform can be treated similarly, but here, scale by scale.The Fourier transform of $W T_{x(t)}(a, \tau)$ is $\overline{W T_{x(t)}(a, \omega)}$. The Fourier transform of $\psi(\tau)$ is $\Psi(\omega)$. By the nature of the Fourier Transform, the Fourier transform of

$$
\begin{aligned}
& \psi_{a, t}(\tau)=\frac{1}{\sqrt{a}} \psi\left(-\frac{\tau-t}{a}\right) \\
& \Psi_{a, t}(\omega)=\left(\sqrt{a} \Psi(a \omega) e^{-i \omega t}\right)^{*}=\sqrt{a} \Psi^{*}(a \omega) e^{i \omega t}
\end{aligned}
$$

By Parseval Theorem, the frequency domain of formula 4 can be expressed as

$$
\begin{aligned}
x(t) & =\frac{1}{c_{\psi}} \int_{0}^{\infty} \int_{-\infty}^{\infty} W T_{x(t)}(a, \tau) \frac{1}{\sqrt{a}} \psi\left(\frac{t-\tau}{a}\right) \frac{d \tau d a}{a^{2}} \\
& =\frac{1}{c_{\psi}} \int_{0}^{\infty} \int_{-\infty}^{\infty} W T_{x(t)}(a, \tau) \frac{1}{\sqrt{a}} \psi\left(-\frac{\tau-t}{a}\right) \frac{d \tau d a}{a^{2}} \\
& =\frac{1}{c_{\psi} a^{3 / 2}} \int_{0}^{\infty} \frac{d a}{2 \pi} \int_{-\infty}^{\infty} \frac{W T_{x(t)}(a, \omega) \Psi^{*}}{(a \omega) e^{i \omega t} d \omega}
\end{aligned}
$$

It is obvious that both formulas (6) and (8) contain the basic form of inverse fourier transform, so the inverse discrete fourier transform can be used for numerical processing.
The wavelet transform modulus Maxima of the signal can identify the sudden change of the signal, which is based on the following two points:

1 ) Assume $\theta(t)$ is a low-pass smooth function, the signal $x(t)$ can obtain $y(t)$ after $\theta(t)$ smoothing, and then take the derivative of $y(t)$, equivalent to the direct use derivatives of $\theta(t)$ for signal processing (such as convolution) .

2 ) Any low-pass smooth function $\theta(t)$, its derivatives must be bandpass functions, the frequency characteristic is that there must be a zero point at $\omega=0$, the admissible condition of wavelet transform is satisfied, Therefore, the derivative of $\theta(t)$ can be used as a basic wavelet 。

In summary, if we take the first derivative of a low-pass smooth function $\theta(t)$ as the Basic Wavelet $\psi(t)$, it is used as a wavelet transform for signal $x(t)$, where the zero of the wavelet transform is the point of $\frac{d y}{d t}=0$, that's the extreme point of $y(t)$; The extreme point of the wavelet transform is where $\frac{d^{2} y}{d t^{2}}=0$, the turning point of $y(t)$, is the step point under the limit case (step). Same thing on multiple scales.

In addition, Mallat and Hwang have shown that if the wavelet transform of a signal does not have a modulus maximum at a small scale, then the signal must be locally regular. It can be seen that the modulus maximum of wavelet transform is closely related to the abrupt change of signal. But this does not mean that the modulus Maxima of wavelet transform must correspond to the signal mutation, Mallat proves this point by a counter-example, and also puts forward the corresponding method to solve this problem, the Lipschitz Index, which describes the regularity of the curve, is used to further verify the detected "mutation", thus ensuring the feasibility of identifying the signal mutation with wavelet transform modulus Maxima.

The Lipschitz index $\alpha$ can quantitatively describe the local characteristics of the signal: When $\alpha$ is negative, the point changes dramatically, if $\alpha=-1$ of the $\delta$ function, $\alpha=-0.5-\varepsilon, \varepsilon>0$ of the white noise; ; The larger $\alpha$ is, the more regular the point is. If $0 \leq \alpha \leq 1$ is the signal that is discontinuous at that point but has a finite value, such as $\alpha=0$ for step functions, $\alpha=1$ for ramp functions, and $\alpha>1$ for derivative at that point. 
Mallat proves that the function $x(t) \in L^{2}(R)$ is uniformly Lipschitz $\alpha$ in the $t \in\left[t_{1}, t_{2}\right]$ interval, If and only if $x(t)$ satisfies the wavelet transform under the scale $a$

$$
\left|W T_{x(t)}(a)\right| \leq k a^{\alpha}
$$

$$
\begin{aligned}
& \text { I. E } \\
& \log \left|W T_{x(t)}(a)\right| \leq \log k+\alpha \log a
\end{aligned}
$$

$k$ is a constant in the formula.

Formula (10) shows that if LipschitzIndex $\alpha>0$ of the signal at a certain point, its wavelet transform modulus will increase with the increase of scale. On the contrary, if its Lipschitz Index $\alpha<0$, then its wavelet transform modulus will decrease with the increase of the scale. For the step case $(\alpha=0)$, the wavelet transform Modulus of the signal does not change with the scale.

In summary, the rule of signal and white noise changing with wavelet transform scale is different and the rule of some special types of sudden change and normal signal changing with wavelet transform scale is also different. In the proper scale range of wavelet transform, the wavelet transform modulus of normal signal will increase with the increase of scale, however, the wavelet transform modulus such as white noise and spike pulse will decrease or even disappear as the scale of wavelet transform increases. Therefore, tracking the final direction of the modulus maximum of the signal wavelet transform from the large scale to the small scale, that is, searching the modulus maximum curve of the signal wavelet Transform, as long as the scale selection is appropriate, the final modulus maximum position may be the signal mutation point or the signal trend mutation point; the modulus maximum curve obtained by the search is the signal rather than the noise of the wavelet transform mode of the link. Therefore, all the wavelet transform corresponding to all the modulus maximum curves can be used to reconstruct the signal details and filter the noise, then, all the wavelet transform on other large scale without searching modulus maximum curve is used to recover the trend information of the signal, and the whole signal can be reconstructed by adding the two. It should be pointed out here that the starting scale of modulus maximum curve search is not the maximum scale of signal wavelet transform, because the wavelet function of larger scale has longer duration and the signal wavelet transform is smoother, the wavelet transform of larger scale is the reflection of the signal trend, and the existence of larger scale is only to improve the accuracy of signal reconstruction.
It can be seen that this method can detect the abrupt change of the signal and filter the singularity such as noise or spike pulse, so as to realize the signal reconstruction.

\section{Multi-information fusion method based on evidence theory}

The evidence theory uses the recognition framework $U$ to represent the set of propositions of interest, which defines the basic probability assignment function ( BPAF) over $U$ $m: 2^{U} \rightarrow[0,1]$, satisfy

$$
\left.\begin{array}{c}
m(\Phi)=0 \\
\sum_{A \subset U} m(A)=1
\end{array}\right\}
$$

In the formula, proposition $\mathrm{A}$ is a non-empty subset of $U$, and $M(A)$ reflects the reliability of A.

If $\mathrm{K}$ is the BPAF derived from $\mathrm{n}$ independent evidences on the same recognition frame $U$, then the combination rule of Dempster is used to calculate the BPAF under the action of these independent evidences.

$$
m(C)=\left\{\begin{array}{cc}
0, & C=\Phi \\
\prod_{\bigcap_{i}=A} \prod_{i=1}^{n} m_{i}\left(A_{i}\right) & \\
1-\sum_{\bigcap_{i}=\Phi} \prod_{i=1}^{n} m_{i}\left(A_{i}\right) &
\end{array}\right.
$$

How to make a decision after using evidence theory is a problem closely related to its application. The common decision-making methods include decision-making based on trust function, decisionmaking based on assignment of basic probability and decision-making based on minimum risk. Because the computation is small, the decision method based on the basic probability assignment is adopted here. Let $\mathrm{U}$ be the identification frame. $\mathrm{M}$ is the basic probability assignment based on Dempster combination rule. assume $\exists A_{1}, A_{2} \subset U$,satisfy

$$
\left.\begin{array}{c}
m\left(A_{1}=\max \left\{m\left(A_{i}\right), A_{i} \subset U\right\}\right. \\
m\left(A_{2}=\max \left\{m\left(A_{i}\right), A_{i} \subset U \text { 且 } A_{i} \neq A_{1}\right\}\right)
\end{array}\right\}
$$

if

$$
\left.\begin{array}{c}
m\left(A_{1}\right)-m\left(A_{2}\right)>\varepsilon_{1} \\
m(u)<\varepsilon_{2} \\
m\left(A_{1}\right)>m(U)
\end{array}\right\}
$$

then $A_{1}$ is the verdict. 
In the formula, $m(u)$ represents an unknown fundamental probability; $\varepsilon_{1}, \varepsilon_{2}$ is a predetermined threshold.
Firstly, an Agent-oriented modeling method and a distributed fault monitoring model are used to build the system structure of the intelligent fault monitoring system, as shown in figure 1 .

\section{Example analysis}

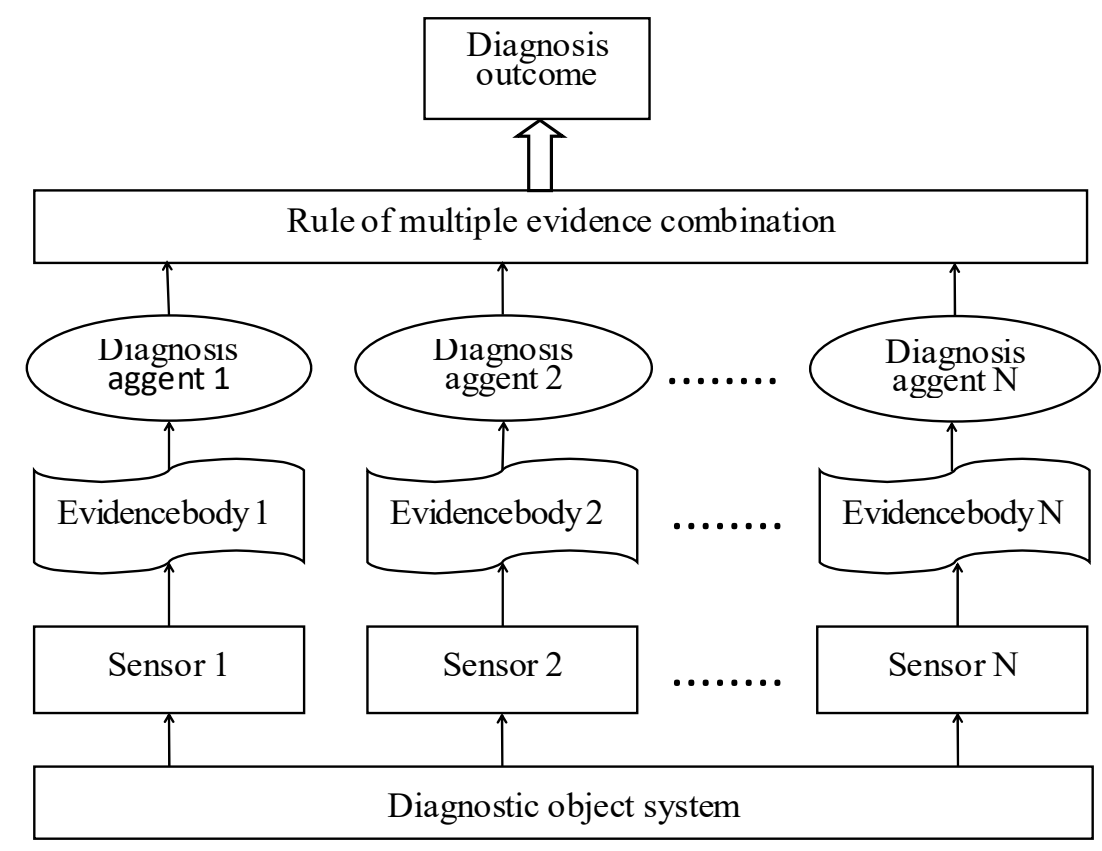

Figure 1. Structure of fault monitoring system based on evidence theory.

Four evidence bodies are used to analyze the fault monitoring capability of the fault monitoring system. The basic probability distribution of $\mathrm{F}_{1}$ (fault A), $F_{2}$ (fault B) and $F_{3}$ (Fault $C$ ) in the fault

Table 1 . The basic probability distribution of the four evidence bodies.

\begin{tabular}{ccccc} 
Evidence & $\mathrm{F}_{1}$ & $\mathrm{~F}_{2}$ & $\mathrm{~F}_{3}$ & $\Theta$ \\
\hline $\mathbf{E}_{\mathbf{1}}$ & 0.98 & 0.01 & 0.01 & 0 \\
$\mathbf{E}_{\mathbf{2}}$ & 0 & 0.01 & 0.99 & 0 \\
$\mathbf{E}_{\mathbf{3}}$ & 0.90 & 0.01 & 0.09 & 0 \\
$\mathbf{E}_{\mathbf{4}}$ & 0.90 & 0.01 & 0.09 & 0 \\
\hline
\end{tabular}

Since the evidence bodies E1 and E2 are in conflict with each other, the use of the rule of evidence composition will produce results contrary to the facts, the combination rule of evidence theory based on support degree of Focus Element can space is obtained from the preliminary diagnosis analysis of four evidence bodies, as shown in Table 1 .

Table 2. The composite of the four evidence bodies.

\begin{tabular}{cccccc}
\hline Evidence & $\mathrm{F}_{1}$ & $\mathrm{~F}_{2}$ & $\mathrm{~F}_{3}$ & $\Theta$ & $\begin{array}{c}\text { Diagnostic } \\
\text { conclusion }\end{array}$ \\
\hline $\mathbf{E}_{\mathbf{1 2}}$ & 0.98 & 0.01 & 0.01 & 0 & $\mathrm{~F}_{3}$ \\
$\mathbf{E}_{\mathbf{1 2 3}}$ & 0 & 0.01 & 0.99 & 0 & $\mathrm{~F}_{2}$ \\
$\mathbf{E}_{\mathbf{1 2 3 4}}$ & 0.90 & 0.01 & 0.09 & 0 & $\mathrm{~F} 1$ \\
\hline
\end{tabular}

As can be seen from Table 2, the new composition rule can reallocate conflicts according to the degree of Focus Element's support for conflicting evidences. In the previous example, the effectively avoid the influence of conflicting evidence on the combination result. The composite results for the four evidence bodies are shown in Table 2.

diagnosis was $\mathrm{F}_{3}$ when there were only two conflicting sources of evidence, but with the addition of new evidence to support $F_{1}$, the diagnosis became $F_{1}$, this shows that the new 
combination rule can not only avoid conflict effectively, but also reflect the influence of new evidence body on the diagnosis result quickly, and has higher diagnosis efficiency.

\section{Conclusion}

The multi-information fusion algorithm of equipment fault health monitoring based on evidence theory is based on multi-information monitoring and using Dempster combination rule. Firstly, the attributes of health monitoring characteristic parameters are fused. Secondly, the time domain fusion of multiple monitoring cycles is carried out, and then the spatial domain fusion of multiple monitoring devices is carried out, and finally the fault source is identified. The multiinformation fusion method of equipment structure and moving parts health monitoring based on evidence theory can process abnormal data, and the filtering ratio of noise data and error information is over $90 \%$, multi-information fusion fault source identification reliability $\geq 90 \%$.

\section{References}

1. Dousis D A.Design and implementation of the V-22 Tiltrotor aircraft vibration monitoring and diagnostic sy8tem.0-7803-5846-5

00.200IEEE.2000: 245-26.

2. Prosser W H. Development of structural health management technology for aerospace vehicles[A].NASA LaRc, JANNAF 39th CS / 27th APS / 21st PSHS / 3rd MSS Joint Subcom. meeting, 2003-12.

3. White E V. Progress in structural health management for aerospace Vehicles[R]. Los Alanl06 National Lab Damage Prognosis Workshop, 2001.03.

4. Staszewski W J, Boiler C, Tomlinson G R.Health monitoring of aero-space structures: smart sensor technologies and signal processing[M].England: John Wiley \& Sons Ltd, 2004: 1-3.

5. Park H, Mackey R, James M, Zak M, et al . Analysis of Space Shuttle Main Engine Data Using Beacon-based Exception Analysis for Multi-Missions[J].Proceedings of the IEEE Aerospace Conference, IEEE , 2002: 6-2835-62844.

6. Giurgiutiu V, Zagrai A and Bao Jing Jing - Piezoelectric wafer embedded active sensors for aging aircraft structural health monitoring[J].Structural Health Monitoring,
2002, 1(1): 41-61.

7. Baruah $\mathrm{P}$, Chinnam RB, HMMs for diagnostics and prognostics in machining processes. Proc. of the 57th Society for Machine Failure Prevention Technology Conference Virginia Beach,VA, 14-18 April.

8. http://www.calce.umd.edu/whats_new/2005/PH $\mathrm{M} / \mathrm{phm} . \mathrm{htm}$.

9. Isermann R.Model-based fault-detection and diagnosis-status and applications[J].Annual Reviews in Control,2005,29(1):71-85.

10.Coker David W and Hallinan J Gary. A logistician's primer on GCSSArmy $(\mathrm{PLM}+)[\mathrm{J}]$. Army Logistician, 2006, 38(3): $35-37$. 